\title{
DESIGN OF CHERRY-PICKING MANIPULATOR BASED ON PLC HIGH SPEED PARALLEL AUTOMATION CONTROL
}

\author{
Can Zou ${ }^{1}$ \\ ${ }^{1}$ School Of Electro-Mechanical Engineering,Hangzhou Polytechnic, \\ Hangzhou 311400, Zhejiang, China. \\ Email: zoucanhz0121@163.com
}

\begin{abstract}
In order to reduce labor costs, solve the shortage of labor, improve the efficiency of harvesting and other issues and the performance of the existing mechanical arm, an experimental software platform will be built based on embedded PLC prototype system, and then the control methods of improving the speed and grasping accuracy of the manipulator will be systematically studied based on the dynamics model of the manipulator from three aspects: rigid body dynamics modelling, trajectory planning and dynamic control methods. The integrated optimization design method of multi-variables based on dynamic scale synthesis, dynamic optimization design and optimal trajectory planning is realized. The kinematics, rigid body dynamics and elastodynamics of the system can be guaranteed simultaneously. The kinematics and rigid body dynamics models of the manipulator are established. A dynamic scale synthesis method considering both kinematics and rigid body dynamics characteristics is proposed. Characteristics are formed in dynamic evaluation index and performance constraints. Finally, the results show that the load characteristics and dynamic characteristics of the cherry-picking manipulator based on PLC high-speed parallel automation control are in good agreement with the theoretical values, and the validity of rigid body dynamics and elastic dynamic model is verified. The self-avoidance obstacle of cherry manipulator in coordinated picking is solved, and the singularity of cherry manipulator in picking is avoided. The high speed and barrier-free operation of the manipulator is realized.
\end{abstract}

Keywords: Mechanical Arm; PLC; High-Speed Parallel Automation; Dynamic Model; Embedded.

\section{Introduction}

Soft PLC technology is a new control technology based on PC, which is gradually emerging in the field of international industrial automation. Embedded soft PLC technology is developed on the basis of indepth study and analysis of traditional PLC technology, soft PLC technology and embedded system. Most of the PLC control programs applied to industrial control at present only contain the development status of common basic control instructions [1]. Embedded soft PLC can make up for the shortcomings of traditional PLC, such as poor compatibility and scalability, low cost performance ratio, and overcome the shortcomings of large-scale soft PLC system and waste of resources. The embedded soft PLC system used in this study has the advantages of flexibility, compactness, convenience and reliability. The embedded soft PLC prototype system mainly includes the editing system of ladder diagram of upper computer, serial communication system, instruction interpretation system of lower computer and input and output system. The embedded soft PLC system can well realize the basic functions of traditional PLC system and simplify the instruction interpretation system of PLC through PLC instructions [2].

The control strategy of high-speed parallel automation mainly includes conventional control strategy based on model and intelligent control strategy based on Intelligent algorithm. The conventional control strategy mainly includes adaptive control, robust control, decoupling control, PID control and so on.

The adaptive control method relies less on prior knowledge and can continuously identify the realtime operation state of the manipulator, extract effective model information through strong learning ability, and continuously improve the model [3]. At present, the adaptive control of manipulator is usually based on the dynamic model to separate the relevant physical parameters, then the nonlinear and strong coupling dynamic model is constructed into the linear expression of the parameters to be identified, and then the global convergence adaptive control rate is designed by using the mature adaptive control theory of linear system. Considering the uncertainties of friction and dynamic characteristics, some people choose the parameters of friction model and dynamic model as adaptive control parameters. These adaptive parameters are separated from the 
dynamic model of parallel robot, and corresponding adaptive friction compensation control and adaptive dynamic compensation control are designed.

This study makes use of the characteristics of PLC, such as high reliability, strong anti-interference ability, powerful function, flexibility, easy to learn and use, small size, light weight and low price. A high-speed parallel automatic control cherry picking manipulator based on PLC is designed to make the manipulator have better operation performance.

\section{Method}

In order to solve the problem of low efficiency of cherry-picking robot with single manipulator, a highspeed parallel automated cherry-picking scheme is determined. Firstly, the three-dimensional coordinates of cherries are obtained by Kinect sensor, and the three-dimensional coordinates of cherries are transmitted to the manipulator, which picks cherries. The machine vision system divides the identified cherries into two regions. The robot arm adopts the proximity principle and picks the cherries in the adjacent regions. Aiming at the problem that the end effectors is easy to collide with cherries outside the target fruit, a sequential rule for cherry picking by mechanical arm is proposed. When the manipulator picks adjacent kiwifruit, it solves the self-avoidance obstacle of coordinated picking of cherry manipulator, so that the two manipulators do not interfere with each other when picking kiwifruit. At the same time, the path planning of the manipulator is carried out in Cartesian space and the singularity of the manipulator when picking cherry is avoided.

The difficulties of integrated optimization design of high-speed parallel manipulators can be expressed as follows. Firstly, the models related to the design of high-speed parallel manipulators include kinematics, rigid body dynamics and elastic dynamics models.

The design parameters (scale, cross-section, material) and performance indicators involved are numerous, which brings some difficulties to the design [5]. Secondly, in order to satisfy the performance indexes of high speed, high acceleration, accuracy and power consumption in kinematics, rigid body dynamics and elastodynamics, there is no design process that can guide crossmodel optimization.
In order to solve the above problems and achieve the purpose of energy saving and consumption reduction, from the point of view of a kind of highspeed parallel manipulator system, various factors affecting the above performance indexes of the manipulator are considered comprehensively in terms of input, system itself and output.

As far as system ontology is concerned, the main task of its design is to determine a set of scales and structural parameters that meet the performance requirements of the system. Considering the different theoretical foundations of kinematics, rigid body dynamics and elastodynamics, the design idea is to divide the scale synthesis and dynamic design into two different design dimensions by using hierarchical strategy. Then, numerical methods are used to unify the above optimization results. Before optimizing the main body of high-speed parallel manipulator, the relative reducer and deceleration ratio should be selected according to the given design task and motor rated speed.

Then, scale synthesis is completed at the level of kinematics and rigid body dynamics, that is, given the structural parameters $\mathrm{X}_{20}$ in advance. By investigating the influence of scale parameters on the dynamic evaluation index under the constraint of kinematics performance, the optimal scale parameters considering both kinematics and dynamic performance under the specific structural parameters $X_{1}$ are synthesized. $X_{1}$ is taken as input, and the dynamic optimization design satisfying dynamic accuracy constraints is completed at the level of elastodynamics [6]. After obtaining the optimal structural parameters $\mathrm{X}_{2}$, the structural parameters $\mathrm{X}_{2}$ are fed back to the scale synthesis to realize the closed-loop design until the judgment conditions are satisfied.

Rigid body dynamics modeling: There are two purposes of rigid body dynamics modeling. One is to evaluate the dynamic performance of the manipulator, and the other is to provide the necessary mathematical model for the implementation of torque control and parameter estimation of servo motor. On the basis of the velocity and acceleration model of the follower arm branched chain, connecting coordinate system $\mathrm{O}_{\mathrm{bi}}-\mathrm{x}_{\mathrm{bi}} \mathrm{y}_{\mathrm{bi}} \mathrm{z}_{\mathrm{bi}}$ is established on the follower link of the it branch chain.

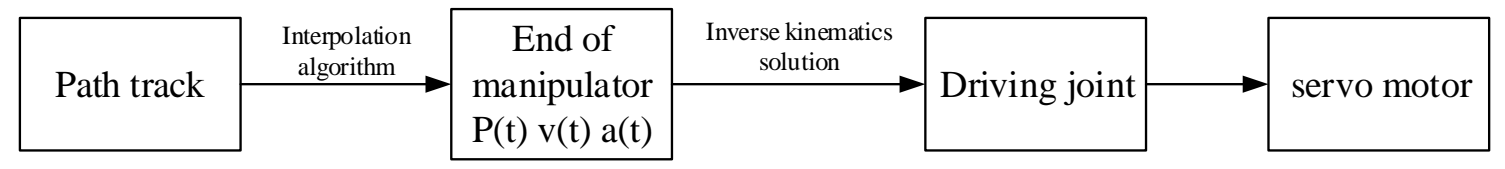

Figure 1: Trajectory planning in operation space 


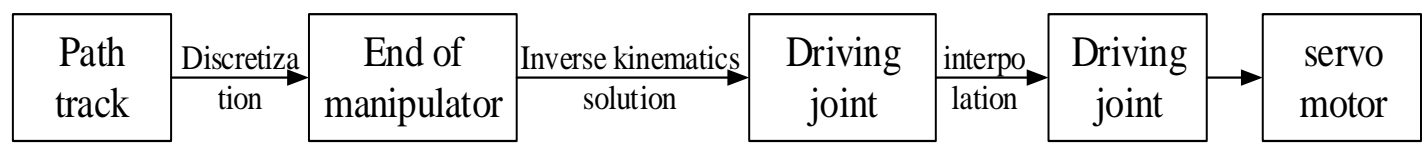

Figure 2: Joint space trajectory planning

The coordinate origin $\mathrm{O}_{\mathrm{bi}}$ is located on the center of mass of the branch chain, and the $\mathrm{Z}_{\mathrm{bi}}$ axis always points to the axis direction of the connecting rod. The $\mathrm{x}_{\mathrm{bi}}$ axis is perpendicular to the $\mathrm{z}_{\mathrm{bi}}$ axis. In the plane of $\mathrm{w}_{\mathrm{bi}}$ and $\mathrm{v}_{\mathrm{i}} \times \mathrm{u}_{\mathrm{i}}$, the $\mathrm{y}_{\mathrm{bi}}$ axis is perpendicular to $\mathrm{X}_{\mathrm{bi}}$ and $\mathrm{z}_{\mathrm{bi}}$ axis, and conforms to the right-hand criterion. The dynamic equation of the mechanism can be obtained by using the principle of virtual work.

$$
\begin{aligned}
& \delta \dot{\theta} \tau^{\prime}= \\
& \delta \dot{\theta}^{\mathrm{T}}\left(\mathbf{I}_{\mathrm{A}}^{\prime} \ddot{\boldsymbol{\theta}}+\tau_{\mathrm{Ag}}^{\prime}\right)+ \\
& \sum_{i=1}^{3}\left(\delta \delta_{i}^{\mathrm{T}} \mathbf{m}_{\mathrm{si}}+\delta r_{i}^{\mathrm{T}} \dot{f}_{\mathrm{si}}\right)+ \\
& \delta \dot{\mathbf{r}}^{\mathrm{T}}\left(\mathbf{m}^{\prime} \ddot{\mathbf{r}}+\mathbf{m}^{\prime} \mathbf{g} \hat{z}\right)
\end{aligned}
$$

In the formula, $\tau=\left(\begin{array}{cccc}\tau_{1} & \tau_{2} & \tau_{3}\end{array}\right)^{\mathrm{T}}$ is the torque of the active joint; $\ddot{\mathrm{r}}$ is the acceleration of the center of mass of the moving platform; $m$ ' is the total mass of the moving platform, including the mass of the platform and the load; $\mathrm{I}_{\mathrm{A}}$ is the equivalent inertia of the active arm to its axis, that is, $\mathrm{I}_{\mathrm{A}}^{\prime}=\mathrm{I}_{\text {arm }}+\mathrm{j}^{2} \mathrm{I}_{\text {gearbox }}$, where $\mathrm{I}_{\mathrm{arm}}$ is the inertia of the active arm itself to its axis, $I_{\text {gearbox }}$ is the inertia of the reducer, and $\mathrm{j}$ is the reduction ratio of the reducer. The moment of gravity of the active arm to its rotating shaft is: $\tau_{\mathrm{Ag}}^{\prime}=\mathrm{m}_{\mathrm{arm}} \mathrm{r}_{\mathrm{arm}} \mathrm{g}\left(\begin{array}{lll}\cos \theta_{1} & \cos \theta_{2} & \cos \theta_{3}\end{array}\right)^{\mathrm{T}}$.

$\mathrm{m}_{\mathrm{A}} \mathrm{r}_{\mathrm{A}}$ is the mass-diameter product of the active arm. The motion quantities represented by independent variational $\delta \dot{\theta}$ are substituted into equation 1.

$$
\begin{aligned}
& \tau= \\
& \left(\mathrm{I}_{\mathrm{A}} \ddot{\theta}+\tau_{\mathrm{Ag}}{ }^{\prime}\right)+ \\
& 1_{1} \mathrm{~J}^{-\mathrm{T}}\left[\sum_{\mathrm{i}=1}^{3}\left(\mathrm{~J}_{\mathrm{wi}}^{\mathrm{T}} \mathrm{m}_{\mathrm{si}}+\mathbf{J}_{\mathrm{vi}}^{\mathrm{T}} \mathrm{f}_{\mathrm{si}}\right)+\left(\mathrm{m} \cdot \ddot{\mathrm{r}}+\mathrm{m}^{\prime} \mathrm{g} \hat{\mathrm{z}}\right)\right]
\end{aligned}
$$

Trajectory planning: The dynamic characteristics of the high-speed parallel automatic control manipulator are reflected in the track tracking accuracy and positioning accuracy written in highfrequency motion conditions to a certain extent. The trajectory tracking accuracy and positioning accuracy are related not only to the characteristics of the manipulator body and the control system [7], but also to the trajectory of the manipulator end effectors in the operating space and the different motion rules selected. Therefore, reasonable trajectory planning can effectively improve the operation accuracy and dynamic characteristics of high-speed parallel automatic control manipulator.

According to the choice of planning space, the trajectory planning of manipulator can be divided into operation space trajectory planning and joint space trajectory planning. In contrast, the trajectory planning in the operating space has clear trajectory, which can strictly ensure that the end of the actual manipulator moves according to a specific trajectory. It is suitable for situations with obstacle avoidance requirements and continuous trajectory control. In addition, there are singular points in the motion process of the method, which need to be checked and avoided in the trajectory planning.

The trajectory planning process of operation space and joint space is shown in figure 1 and figure 2 above. Considering both the operating space and the joint space, considering the minimum maximum acceleration of the manipulator and reducing the vibration of the manipulator, a trajectory planning method which satisfies the requirements of Pick amp, and has smooth process, low energy consumption and simple operation is of great practical significance for the optimization of the dynamic characteristics of the manipulator and the realization of real-time dynamic control.

Table 1. Motor model parameters

\begin{tabular}{|c|c|c|c|}
\hline Variable coincidence & Meaning & numerical value & unit \\
\hline $\mathrm{K}_{\mathrm{pi}}$ & $\begin{array}{c}\text { Proportional Coefficient of Current } \\
\text { Loop }\end{array}$ & 55 & $\mathrm{~V} \cdot \mathrm{A}^{-1}$ \\
\hline $\mathrm{T}_{\mathrm{ii}}$ & Integral time constant of current loop & 10 & $\mathrm{~S}$ \\
\hline $\mathrm{K}_{\mathrm{t}}$ & Motor Torque Constant & 1.345 & $\mathrm{~N} \cdot \mathrm{m}^{-1} \mathrm{~A}^{-1}$ \\
\hline $\mathrm{K}_{\mathrm{e}}$ & Motor Back EMF Constant & 1.002 & $\mathrm{~V} \cdot \mathrm{s} \cdot \mathrm{rad}^{-1}$ \\
\hline $\mathrm{L}$ & Inductance of motor winding & 39.995 & $\mathrm{H}$ \\
\hline $\mathrm{R}$ & Motor winding resistance & 6.098 & $\Omega$ \\
\hline $\mathrm{J}$ & Rotary inertia of motor rotor & $3.4^{*} 10^{-4}$ & $\mathrm{Kg} \cdot \mathrm{m} 2$ \\
\hline
\end{tabular}


Dynamics control method: At present, one of the commonly used dynamic control methods is the dynamic feed-forward control method, which takes a single branch chain of the mechanism as the controlled object, uses the dynamic model and realtime kinematics parameters to solve the theoretical control moment, and then compensates the dynamic signal to the kinematics closed-loop system. This method retains the position control accuracy of kinematics closed-loop control, and the kinematics closed-loop system has good resistance, which reduces the dependence on the accuracy of dynamic model modelling [8]. This dynamic feedforward control method with single branched chain as the research object is simple in principle and easy to implement. It has certain popularization value for parallel mechanism. Therefore, the high-speed parallel automatic control manipulator is taken as the research object, and the control effect of dynamic control based on PLC on parallel mechanism is discussed.

The model of servo control system is built from inside to outside. Firstly, the control model of servo motor is established. The schematic diagram of armature control of servo motor is given, as shown in figure 3.

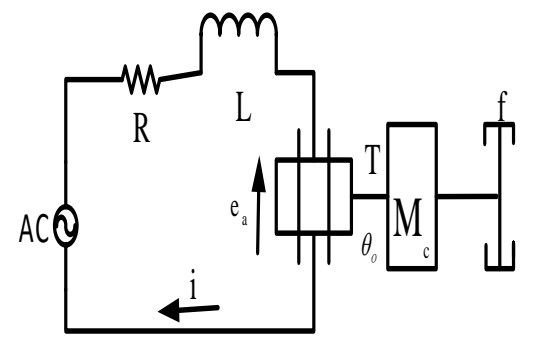

Figure 3: Schematic diagram of servo motor principle

The motor model selected in this study is LAFERT B7104Q3H7GKM00T5. The servo driver adopts the CoolDriveA8 Book motor drive module of Qingneng Dechuang. By consulting the Lafayette Motor Selection Manual and the configuration parameters of Qingneng Dechuang, the main parameters of current loop and motor model can be obtained as shown in table 1.

Usually, the current loop servo parameters of the servo control system are not open to users, and only the proportional coefficient $\mathrm{K}_{\mathrm{iv}}$ and integral time constant of the speed loop $\mathrm{T}_{\mathrm{iv}}$, as well as the proportional coefficient $\mathrm{K}_{\mathrm{ip}}$ and integral time constant of the position loop $\mathrm{T}_{\mathrm{ip}}$ in the model are needed.

Differential time constants $T_{d p}$ need to be tuned according to the actual model of the control system to achieve good control effect of closed-loop kinematics control.

\section{Results and Discussion}

The automatic parallel operation device changes the previous method of automatic parallel operation using pulsating voltage. Frequency detection, voltage detection and phase detection all adopt digital detection method. The frequency of the generator is detected by the photoelectric encoder connected with the generator coaxially, and then the frequency difference can be obtained by calculating. The direction of frequency difference can be directly reflected by the detected frequency. Detection is obtained directly by comparing with standard voltage, and phase detection is obtained by edge detection of voltage square wave. The control of automatic parallel operation can be realized by analog control device or digital control device.

This device is a high-speed automatic parallel operation device of manipulator arm controlled by PLC. The automatic parallel operation device of PLC also has the advantage of automatic parallel operation device controlled by computer, but it is superior to computer control in working reliability. It can automatically detect the three conditions of parallel operation. When the voltage, frequency and phase all meet the conditions of parallel operation, it can issue the closing instructions accurately and realize the function of automatic parallel operation. The block diagram of each performance test for the manipulator is shown in figure 4.

The control program is programmed with modular design idea. The automatic parallel operation function of the manipulator is studied. Then the modules are divided according to the results of the study. The main modules are linked to each other to realize the control of the automatic parallel operation.

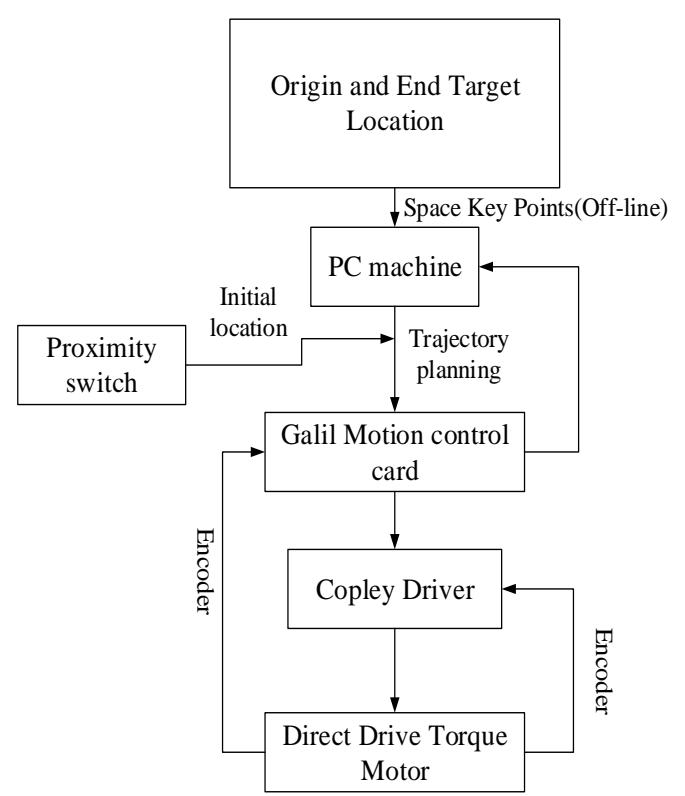

Figure 4: Structure diagram of manipulator control system 
The automatic parallel operation subsystem has the function of automatically analyzing and judging the current state of the manipulator arm.

Therefore, some state variables are set up in the subsystem. In the main module program, the PLC continuously reads, synthesizes and analyses these state variables, and decides which processing module to call according to the judgment result. Once the module is executed, it also decides which processing module to call.

Returning to the main program and continuing to cycle the detection of state variables, the control device will continue to work in such a continuous cycle.

\section{Conclusion}

The application of PLC in the control of manipulator arm and the realization of automatic frequency and load regulation in operation process is a new development and supplement of automatic frequency and load regulation control technology. It has the advantage that traditional analog control scheme cannot achieve. Compared with the automatic frequency and load regulation device controlled by computer, the two methods have their own advantages. However, the reliability of PLC control is better than that of computer control, so it is more suitable for the application of auto-control manipulator arm. Through the research on the PLC automatic frequency and load regulation device of high-speed parallel automatic control manipulator arm, the use of PLC for automatic frequency and load regulation control are fully understood.

Firstly, the use of PLC control is a good digital control scheme of automatic frequency modulation and load regulation. It has high control accuracy, rapid response and stable operation, and can fully meet the performance index of automatic frequency modulation and load regulation control of manipulator arm.

Secondly, with advanced PLC as the control core, the reliability of the control is greatly enhanced, and the anti-interference ability is strong. Under the harsh environment, the automatic frequency modulation and load regulation operation can be carried out reliably and accurately, which ensures the continuity and safety of the power supply of the manipulator arm.

Thirdly, the mechanical arm PLC automatic frequency and load regulation device has few components, low cost, high performance-price ratio, has good practical application value, can be used in practice, and has broad market prospects.

In this era of rapid development of information, science and technology are changing with each passing day.

The integrity of the design performance of cherry-picking manipulator based on PLC high-speed parallel automatic control is worthy of more indepth and detailed study.

\section{Reference}

[1] Zhao, D. H., Zhang, H., Hou, J. X. (2014). Design of Fruit Picking Device Based on the Automatic Control Technology. Key Engineering Materials, 620, 7.

[2] Natal, G. S. (2016). Nonlinear control of parallel manipulators for very high accelerations without velocity measurement: stability analysis and experiments on Par2 parallel manipulator. Robotica, 34(1), 43-70.

[3] Dong, L., Song, Y., Tao, S., et al. (2016). Optimum design of a novel redundantly actuated parallel manipulator with multiple actuation modes for high kinematic and dynamic performance. Nonlinear Dynamics, 83(1-2), 631658.

[4] Kazemi, S., Kharrati, H. (2017). Visual Processing and Classification of items on Moving Conveyor with Pick and Place Robot using PLC. Intelligent Industrial Systems, 3(1), 15-21.

[5] Tai, H., Kostromytska, O., Kenworthy, K. E., et al. (2014). Zoysiagrass Genotype Responses to Sphenophorus venatus vestitus (Coleoptera: Curculionidae). Journal of Economic Entomology, 107(4), 1535-42.

[6] Edqvist, P. H., Fagerberg. L., Hallstrã đM, B. M., et al. (2015). Expression of human skin-specific genes defined by transcriptomics and antibodybased profiling. Journal of Histochemistry \& Cytochemistry, 63(2), 129-141.

[7] Dong, L., Song, Y., Tao, S., et al. (2016). Optimum design of a novel redundantly actuated parallel manipulator with multiple actuation modes for high kinematic and dynamic performance. Nonlinear Dynamics, 83(1-2), 631658.

[8] Lang, X., Jin, D., Zhang, C., et al. (2013). Control System Design of DELTA Parallel Manipulator Based on Real-time Ethernet. Robot, 35(5), 576. 